\title{
CONDIÇÕES ADVERSAS NA VIDA DE CRIANÇAS COM ATRASO NO DESENVOLVIMENTO*
}

\author{
ADVERSE CONDITIONS IN THE LIFE OF CHILDREN WITH DEVELOPMENTAL RETARDATION
}

Sônia Santa Vitaliano Graminhaํ \& Maria Angélica de Oliveira Martins²

Docente $^{1}$; Psicóloga do Centro de Psicologia Aplicada ${ }^{2}$ do Departamento de Psicologia e Educação da Faculdade de Filosofia, Ciências e Letras de Ribeirão Preto da Universidade de São Paulo ${ }^{1,2}$.

CoRRESPONDÊNCIA: Departamento de Psicologia e Educação da Faculdade de Filosofia, Ciências e Letras de Ribeirão Preto - Campus Universitário - CEP: 14049-900 - Ribeirão Preto - SP

GRAMINHA SSV \& MARTINS MA de O. Condições adversas na vida de crianças com atraso no desenvolvimento. Medicina, Ribeirão Preto, 30: 259-267, abr./jun. 1997.

RESUMO: O objetivo deste estudo foi o de identificar os fatores de risco a que foram expostas as crianças portadoras de atraso no desenvolvimento, encaminhadas ao Centro de Psicologia Aplicada da FFCLRP-USP, nos últimos cinco anos. O grupo ficou constituído por doze meninos e dez meninas, cujas idades variavam de um mês a oito anos. Os dados foram extraídos das entrevistas realizadas com as mães, no momento da inscrição da criança junto ao serviço. Os problemas referidos pelas mães, na queixa, bem como os eventos e as circunstâncias adversas relatados, na história de vida da criança, foram classificados de acordo com os Sistemas de Categorias elaborados pelas autoras desse estudo. Os resultados mostram que, além do atraso, aparecem referidos na queixa, com maior freqüência, os problemas de fala, coordenação motora, aprendizagem e agitação/inquietude. A análise das circunstâncias adversas, presentes na história de vida desse grupo de crianças, evidenciou que todas elas foram expostas a uma ou mais das condições de risco biológico, sendo as mais freqüentes: problemas congênitos, problemas de saúde física da mãe durante a gestação, problemas de saúde física da criança, crises convulsivas, complicações no parto e hospitalização. Além disso, observou-se que $36 \%$ das crianças havia sido exposta a fatores de risco ambiental, especialmente aqueles ligados a atitudes e práticas de cuidado e educação inadequadas. Com base nos resultados, discutem-se as implicações da presença do risco biológico como condição importante para o acesso da criança e da família a serviços especializados.

UNITERMOS: Fatores de Riscos. Fatores Biológicos. Desenvolvimento Infantil.

\section{INTRODUÇÃO}

O conhecimento dos fatores de risco, que podem levar ao atraso no desenvolvimento, é imprescindível para a implementação de recursos que visem diminuir sua incidência ou minimizar seus efeitos sobre a criança e a família. Que condições levam a criança ao status de risco? Conforme salientam Hanson \&
Lynch, $1989^{1}$, três tipos de condição de risco para atraso no desenvolvimento têm sido tipicamente considerados por estudiosos da área: a de risco estabelecido, que se refere a desordens médicas definidas, especialmente as de origem genética; a de risco biológico, referindo-se a eventos pré, peri e pós-natais, que resultam em dano biológico e que podem aumentar a probabilidade de prejuízo no desenvolvimento, e, final-

\footnotetext{
* Parte deste trabalho foi apresentada no simpósio "Fatores de risco para a saúde mental infantil”, na XXV Reunião Anual da Sociedade Brasileira de Psicologia, outubro de 1995.

Trabalho realizado com apoio financeiro do CNPq.
} 
mente, as experiências de vida ligadas às condições precárias de saúde, à falta de recursos sociais e educacionais, aos estressores familiares e às práticas inadequadas de cuidado e educação, dentre outras, consideradas como integrantes do risco ambiental.

Ao se considerar a relação entre a condição de risco e serviços preventivos, faz-se necessário especificar algumas suposições sobre risco e desenvolvimento. A primeira suposição é a de que o risco não é uma condição única, mas envolve uma variedade de condições e pode assumir graus de severidade variados. $\mathrm{O}$ estudo prospectivo, realizado por Korhonem et al., 1993², teve por objetivo explorar a associação entre fatores de risco perinatais e funções neuropsicológicas avaliadas aos seis anos de idade. Os resultados mostraram que as complicações perinatais severas foram significativamente relacionadas com o resultado do teste $\mathrm{e}$ que as complicações leves não o foram. Assim, o risco perinatal se apresentou em níveis diferentes de severidade e os efeitos sobre o desenvolvimento das crianças foram diferentes. Este mesmo estudo concluiu que o nível educacional baixo da mãe estava significativamente relacionado com a performance neuropsicológica pobre da criança, em $36 \%$ dos casos.

Outra suposição refere-se à visão de que o desenvolvimento está inserido em um contexto de saúde mais amplo. O status de desenvolvimento e outros aspectos de saúde são altamente relacionados. Assim, a má nutrição severa e prolongada é freqüentemente associada com o atraso no desenvolvimento, apatia e dificuldade de concentração; crianças com problemas respiratórios crônicos podem não ter energia e vigor necessários para a participação em atividades educacionais e sociais que podem ser facilitadoras do seu desenvolvimento. Então, a integridade biológica e psicológica são relacionadas e interativas, e devem ser consideradas no planejamento de serviços para crianças com riscos para problemas de desenvolvimento.

A terceira suposição é a de que o desenvolvimento é multideterminado, não sendo, portanto, função somente da suficiência biológica ou ambiental. A genética, a nutrição, a atitude dos adultos e as oportunidades de vivenciar experiências sociais estão entre os vários fatores que contribuem para a saúde física ou psicológica da criança. Pesquisas recentes sobre risco foram guiadas pela hipótese de que fatores biomédicos, associados com o processo de nascimento, estavam diretamente relacionados com conseqüências posteriores de desenvolvimento ${ }^{2 / 6}$. No entanto, a maioria destes estudos demonstrou que a relação entre fatores de risco perinatal e problemas posteriores de desenvolvimento não é direta e que os fatores de risco psicossocial desempenham um papel muito importante. Adams; Hillman \& Gaydos, $1994^{7}$, relatam que a avaliação de crianças na idade pré-escolar se restringia ao desenvolvimento cognitivo com o propósito de determinar a prontidão escolar e avaliar a eficácia dos programas de educação compensatórios, e que esta avaliação se ampliou, incluindo outros domínios do desenvolvimento (comportamental, social, motor, adaptativo), motivada pelo interesse científico no desenvolvimento psicológico do pré-escolar, pelo aumento no número de encaminhamentos para serviços de psicologia, efetuados por professores da pré-escola e pela própria lei pública 99-457 de 1986, que exigia a avaliação da elegibilidade das crianças aos serviços de intervenção precoce. O interesse científico se focalizou na investigação dos fatores de risco presentes no nascimento, que comprometiam o desenvolvimento da criança a longo prazo, predispondo-a a atrasos no desenvolvimento e a problemas emocionais e comportamentais. Assim, os esforços de pesquisa resultaram em listas de variáveis biológicas e ambientais que poderiam estar correlacionadas com o comprometimento posterior do desenvolvimento infantil. Estes autores citam vários estudos, nos quais ficou demonstrado que a combinação de fatores de risco ambiental e biológico coloca uma ameaça dupla no desenvolvimento da criança, e que as influências ambientais têm sido freqüentemente consideradas como os preditores independentes mais importantes dos resultados a longo prazo, em crianças nascidas com fatores de risco médico. Mas, segundo Bendersky \& Lewis, 1994³ , a medida da qualidade ambiental mais comumente usada é o status sócio-econômico, particularmente em estudos de crianças de alto risco, nas quais a conseqüência de condições médicas é o foco de interesse". Os autores alegam que o status sócio-econômico relaciona-se parcialmente com outras variáveis ambientais que, comprovadamente, afetam o desenvolvimento. As variáveis do contexto proximal, tais como a qualidade da interação mãe-criança ou mesmo os brinquedos disponíveis para a criança, apresentam relação com o desenvolvimento precoce. Ao comparar o desenvolvimento, avaliado aos dois anos, através das Escalas Bayley de Desenvolvimento Infantil, de crianças prematuras saudáveis com o de crianças nascidas a termo, Censullo, 19944, encontrou que as crianças prematuras apresentavam um escore significativamente mais baixo no Índice de Desenvolvimento Mental (IDM) do 
que as crianças nascidas a termo. Neste mesmo estudo, a autora avaliou, dentre outros, fatores ambientais proximais, através da aplicação do Home Observation for the Measurement of the Environment (HOME) e distais, como emprego dos pais, mudanças de vida, arranjos para o cuidado da criança, etc. A autora observou que, aos dois anos, as variáveis proximais correlacionavam-se mais fortemente com os escores da Bayley; encontrou correlações moderadas entre subescalas do HOME (responsividade materna e envolvimento) com o IDM da Bayley e correlação baixa entre emprego dos pais e resultados desta mesma escala. Assim, a autora enfatiza que a qualidade do cuidado da criança influencia o seu desenvolvimento posterior; portanto, é importante identificá-la, porque este fator é passível de mudanças, enquanto que, muitas vezes, pouco pode ser feito com relação aos fatores distais.

Thompson et al., $1994^{8}$ também utilizaram os resultados obtidos nas Escalas Bayley, por crianças nascidas com peso muito baixo, considerado como fator de risco biológico, medido através de um escore que se baseava na severidade e duração de processos que causam o dano cerebral. Os autores, com base nos resultados das avaliações realizadas aos seis, quinze e vinte e quatro meses, encontraram que o Índice de Desenvolvimento Mental (IDM) e o Índice de Desenvolvimento Motor (IDP) decresceram no decorrer dos períodos de avaliação. O funcionamento cognitivo e motor variavam significativamente em cada época de avaliação, em função do status de risco biológico, e o funcionamento cognitivo variou, significativamente, em função do risco psicossocial, nas avaliações realizadas aos quinze e vinte e quatro meses. Além disso, as crianças que apresentavam os riscos biológico e psicossocial tiveram os piores resultados motores e cognitivos. Em contraste com os resultados de muitos estudos, o risco biológico teve um impacto contínuo sobre o desenvolvimento, neste grupo de crianças de baixo peso. No entanto, consistentemente com outros estudos, as variáveis sócio-demográficas tornaram-se crescentemente preditivas do desenvolvimento, particularmente do cognitivo, enfatizando a contribuição significante que o estresse materno habitual tem sobre esta área de funcionamento, no decorrer do tempo. Os autores apontam a associação encontrada entre o estresse materno e o baixo nível sócio-econômico, influenciando negativamente o desenvolvimento cognitivo. Os achados deste estudo evidenciaram a ligação entre o estresse materno e o funcionamento cognitivo e psicossocial dos pais e da criança, sugerindo que isto pode servir como um marco dos cuidados do ambiente, que podem maximizar ou minimizar os efeitos da vulnerabilidade biológica.

O que se pode observar, através de vários estudos, é que uma criança pode ser considerada vulnerável, como resultado da exposição a fatores de risco biológico, mas, resistente aos efeitos negativos, na extensão em que seu ambiente forneça experiências compensatórias. Estas informações têm, portanto, implicações importantes para o planejamento de estratégias de intervenção precoce nos problemas de desenvolvimento.

As suposições descritas anteriormente nos levam a considerar a necessidade de uma ligação entre riscos e serviços. O status de risco precoce pode ser identificado e melhorado por serviços apropriados, envolvendo intervenções médicas e sócio educacionais que visem mudar, mesmo que a longo prazo, o impacto da condição de risco sobre a criança. Embora as estratégias para a prevenção do atraso para as crianças do grupo de risco signifiquem o ideal, em termos de atuação, muitas vezes, um serviço de atendimento psicológico, é procurado por pais de crianças pertencentes ao grupo de risco estabelecido ou que tenham sido expostas a condições ambientais adversas que potencializaram os efeitos dos riscos biológicos. Para essas crianças, as estratégias de intervenção deverão ter como objetivo minimizar o impacto da condição de limitação ou prejuízo, promover o seu desenvolvimento e prevenir a ocorrência de problemas de natureza emocional e/ou comportamental associados. Desse modo, a análise das histórias de vida das crianças inscritas num serviço de atendimento psicológico, por estarem apresentando problemas diversos, dentre os quais o atraso no desenvolvimento, possibilita identificar, além da problemática, as condições de risco às quais as crianças foram expostas, fornecendo subsídios para a compreensão dos casos, para a elaboração das intervenções, assim como para suscitar hipóteses que possam ser objeto de novos estudos.

Nesse sentido, o presente estudo tem como objetivo detectar o "perfil de risco" das crianças com atraso no desenvolvimento, encaminhadas para um serviço de atendimento psicológico.

\section{METODOLOGIA}

De um total de trezentas e sessenta e sete crianças, encaminhadas, nos últimos cinco anos, para atendimento psicológico junto ao Centro de Psicologia 
Aplicada (CPA) da FFCLRP-USP, por problemas diversos, foi selecionado, para este estudo, o grupo total de crianças inscritas no serviço com queixa e/ou história de atraso no desenvolvimento. Este grupo ficou constituído por vinte e duas crianças (doze meninos e dez meninas), com idades variando de um mês a oito anos (27\% abaixo de um ano; $23 \%$ entre dois a três anos e $50 \%$ entre seis e oito anos). A escolaridade dos pais é variada, sendo que $50 \%$ é de nível superior; $23 \%$ possui o segundo grau e $27 \%$, um nível equivalente ao primeiro grau. Com relação às mães, $41 \%$ possui nível superior, $23 \%$, o segundo grau e $36 \%$, o primeiro grau.

Os dados foram extraídos das transcrições das entrevistas realizadas com as mães, no momento da inscrição da criança junto ao serviço, conduzidas segundo um roteiro que possibilitava a exploração da problemática da criança, do seu desenvolvimento e funcionamento psicossocial e dos aspectos significativos de seu ambiente e de sua história de vida. As entrevistas, realizadas por profissionais do setor ou por estagiários devidamente treinados e supervisionados, foram gravadas e as fitas integralmente transcritas. Para a identificação dos dados pretendidos, procedeu-se à leitura das transcrições, extraindo os trechos em que os pais faziam referência tanto à problemática que motivou a procura do atendimento quanto aos eventos e às circunstâncias adversas presentes nas histórias de vida das crianças. A seguir, os problemas referidos na queixa, além do atraso no desenvolvimento, assim como os eventos adversos identificados, foram classificados, respectivamente, de acordo com o Sistema de Categorias de Problemas, publicado por Graminha \& Martins, 19949, e com o Sistema de Categorias de Fatores de Risco, publicado por Graminha, Martins \& Miura, $1996{ }^{10}$.

Com base nesta classificação, calculou-se a porcentagem de ocorrência de condições adversas na vida das crianças, assim como a de aparecimento de outros problemas associados ao atraso.

\section{RESULTADOS}

$\mathrm{Na}$ amostra aqui estudada, o atraso no desenvolvimento é referido pelos pais como única queixa em $41 \%$ dos casos. Portanto, para a maioria são relatadas outras dificuldades da criança, além do atraso. Dentre os trinta problemas do sistema de categorias elaborado por Graminha \& Martins, $1994^{9}$, foram referidos, na queixa, juntamente com o atraso de desenvolvimento da criança, apenas os relativos a: agitação/inquietude(AI); problema de fala $(\mathrm{FA})$; problema de aprendizagem(PA); comportamento diferentel estranho $(\mathrm{CD})$; problema de coordenação motora $(\mathrm{CM})$; rebeldia/desobediência/dominância(RD); agressividadelprovocação (AP); problema de eliminação(EL).

A Figura 1 apresenta a porcentagem com que cada um desses problemas apareceu, referido na queixa, junto com o atraso de desenvolvimento.

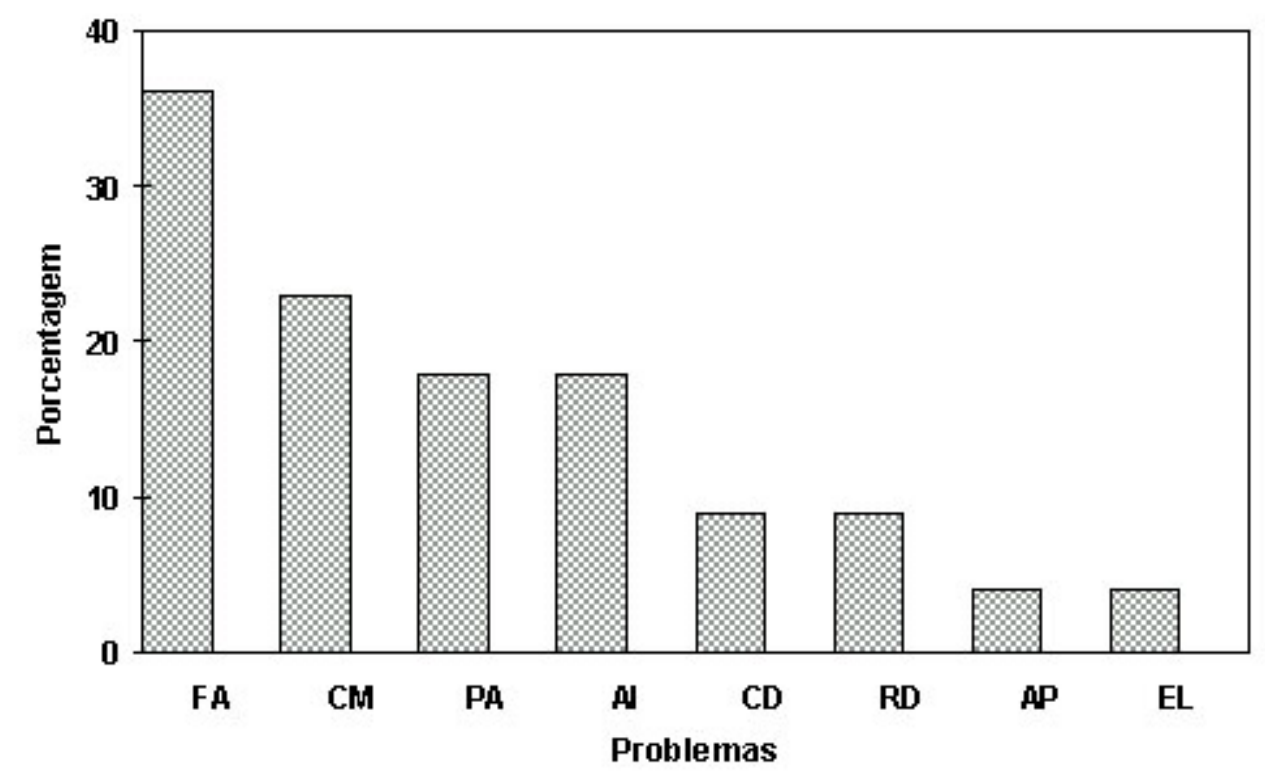

Figura 1 - Porcentagem dos problemas relatados no grupo de crianças com atraso no desenvolvimento. 
Observa-se que os problemas de fala e de coordenação motora são os mais freqüentes $(36 \% \mathrm{e}$ $23 \%$ respectivamente), seguidos de problema de aprendizagem (18\%), agitação/inquietude (18\%), comportamento diferentelestranho(9\%), rebeldia/desobediência/dominância(9\%), agressividade/provocação(4\%) e problema de eliminação( $4 \%)$.

O Sistema de Categorias de Fatores Potenciais de Risco [Graminha \& Martins, 1996 (comunicação pessoal)] utilizado para a classificação dos eventos e condições adversas presentes na história de vida das crianças contêm 34 categorias de fatores, sendo que, na amostra aqui pesquisada, foram identificadas as seguintes: Problemas congênitos $(\mathrm{Pc})$; Problemas de saú- de física da mãe durante a gestação $(\mathrm{Fg})$; Problemas emocionais da mãe durante a gestação (Eg); Complicações no parto $(\mathrm{Cp})$; Prematuridade $(\mathrm{Pr}) ;$ Problemas de saúde física da criança $(\mathrm{Fc})$; Crises convulsivas $(\mathrm{Cc})$; Hospitalização(Hp); Sintomatologia precoce (St); Dificuldades sensoriais(Ds); Baixa auto-estima (Be); Desempenho escolar pobre(De); Gestação na adolescência $(\mathrm{Ga})$; Bebê não desejado (Bñ); Criança adotada (Ca); Dificuldades financeiras da família(Df); Insegurança/indecisão(Is); Superproteção(Su); Indiferença/rejeição(Ir); Agressividade(Ag) e Depreciação(Dp).

A porcentagem de aparecimento de cada um destes fatores de risco, na amostra pesquisada, é apresentada na Figura 2.

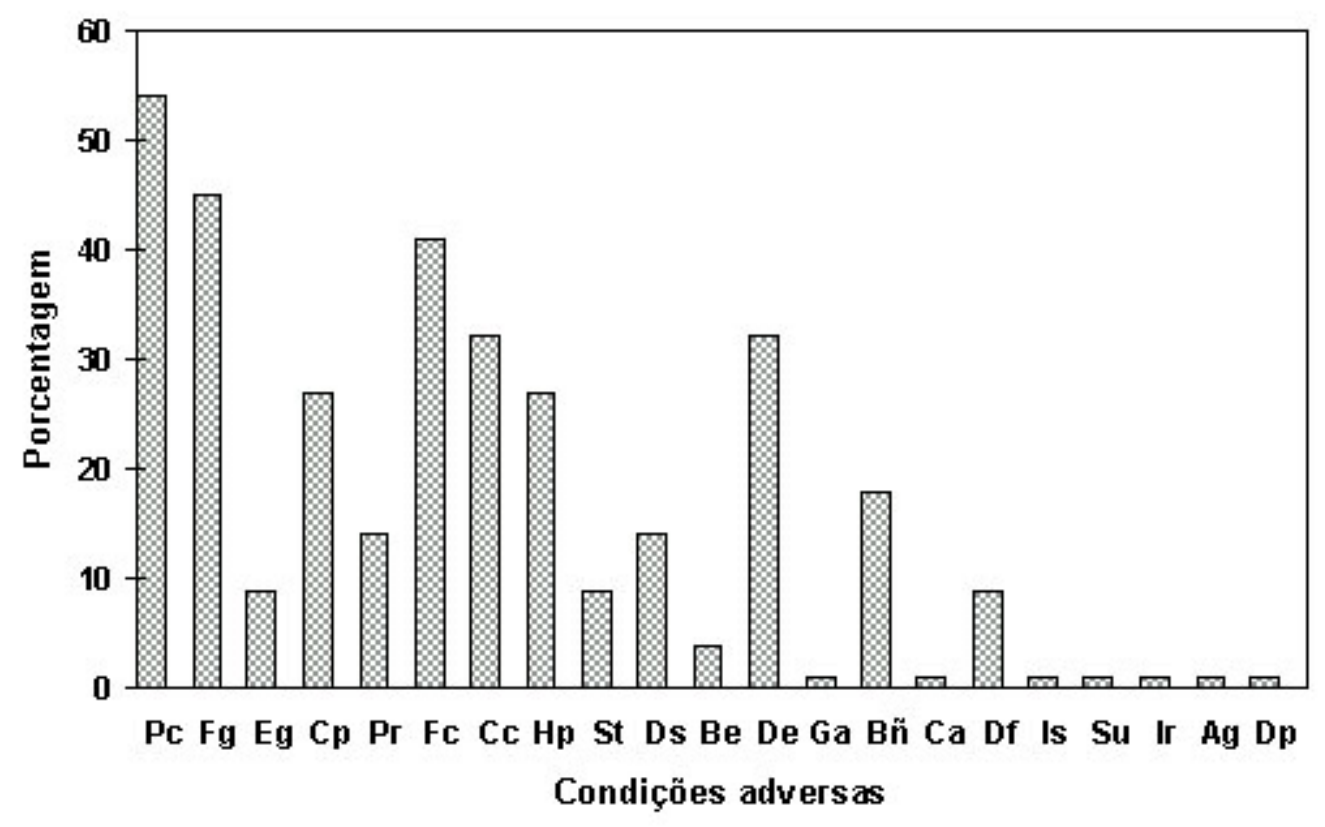

Figura 2 - Porcentagem das condições adversas relatadas no grupo de crianças com atraso no desenvolvimento.

Pode-se observar que a maioria das crianças (54\%) apresentou problemas congênitos, que $45 \%$ das mães entrevistadas teve problemas de saúde física $d u$ rante a gestação, que $9 \%$ delas manifestou problemas emocionais neste mesmo período e $4 \%$ das gestações ocorreram na adolescência da mãe, caracterizando a presença de fatores pré-natais. Dentre os fatores perinatais, as complicações do parto e a prematuridade apareceram em $27 \%$ e $14 \%$ dos casos, respectivamente. Por outro lado, dentre os fatores pós-natais, destacam-se, em primeiro lugar, os problemas de saúde física da criança (41\%), seguidos de crises con- vulsivas e desempenho escolar pobre (32\%), hospitalização(27\%), bebê não desejado(18\%), dificuldades sensoriais específicas(14\%) e sintomatologia precoce e dificuldades financeiras da família (9\%). Embora com incidência mais baixa (4\%), aparecem outros fatores, potencialmente de risco, tais como: baixa auto-estima da criança, criança adotada, insegurança elou inconsistência dos pais, superproteção, indiferença e rejeição, agressividade física elou verbal e depreciação.

Ao serem analisadas as histórias de vida das crianças, constatou-se, na maioria dos casos (73\%), a 
presença de três a sete condições adversas. Procurou-se, então, verificar com que frequiência determinadas condições apareciam associadas, considerando apenas os fatores de maior incidência nesta amostra: problemas congênitos, problemas de saúde física da mãe durante a gestação, complicações no parto, problemas de saúde física da criança, crises convulsivas, hospitalização e desempenho escolar pobre. Pode-se observar que, com exceção do desempenho escolar pobre, as demais categorias referem-se a condições que, de alguma forma, se relacionam com o risco biológico. Os dados são apresentados na Tabela I.

\begin{tabular}{|c|c|c|c|c|c|c|c|c|c|c|c|c|c|c|}
\hline \multirow{3}{*}{ Condições Adversas } & \multirow{2}{*}{\multicolumn{2}{|c|}{$\begin{array}{c}\mathrm{Pc} \\
\mathrm{n}=12\end{array}$}} & \multirow{2}{*}{\multicolumn{2}{|c|}{$\begin{array}{c}\mathrm{Fg} \\
\mathrm{n}=10\end{array}$}} & \multirow{2}{*}{\multicolumn{2}{|c|}{$\begin{array}{c}\mathrm{Cp} \\
\mathrm{n}=6\end{array}$}} & \multirow{2}{*}{\multicolumn{2}{|c|}{$\begin{array}{c}F c \\
n=9\end{array}$}} & \multirow{2}{*}{\multicolumn{2}{|c|}{$\begin{array}{c}\text { Cc } \\
n=7\end{array}$}} & \multirow{2}{*}{\multicolumn{2}{|c|}{$\begin{array}{l}\mathrm{Hp} \\
\mathrm{n}=6\end{array}$}} & \multirow{2}{*}{\multicolumn{2}{|c|}{$\begin{array}{c}\text { De } \\
n=7\end{array}$}} \\
\hline & & & & & & & & & & & & & & \\
\hline & $\mathrm{F}$ & $\%$ & $\mathrm{~F}$ & $\%$ & $\mathrm{~F}$ & $\%$ & $\mathrm{~F}$ & $\%$ & $\mathrm{~F}$ & $\%$ & $\mathrm{~F}$ & $\%$ & $\mathrm{~F}$ & $\%$ \\
\hline Problemas congênitos (Pc) & - & - & 6 & 60 & 3 & 50 & 5 & 55 & - & - & 2 & 33 & 1 & 14 \\
\hline $\begin{array}{l}\text { Problemas de saúde física da } \\
\text { mãe na gestação }(\mathrm{Fg})\end{array}$ & 6 & 50 & - & - & 2 & 33 & 5 & 55 & 3 & 43 & 3 & 50 & 3 & 43 \\
\hline $\begin{array}{l}\text { Problemas emocionais da mãe } \\
\text { na gestação }(\mathrm{Eg})\end{array}$ & - & - & 1 & 10 & - & - & 1 & 11 & 1 & 14 & - & - & 1 & 14 \\
\hline Complicações no parto (Cp) & 3 & 25 & 2 & 20 & - & - & 3 & 33 & 3 & 43 & 2 & 33 & 2 & 29 \\
\hline Prematuridade (Pr) & 1 & 8 & 1 & 10 & 2 & 33 & - & - & 2 & 29 & 1 & 17 & 2 & 29 \\
\hline $\begin{array}{l}\text { Problemas de saúde física da } \\
\text { criança }(F c)\end{array}$ & 5 & 42 & 5 & 50 & 3 & 50 & - & - & 2 & 29 & 4 & 67 & 2 & 29 \\
\hline Crises convulsivas (Cc) & - & - & 3 & 30 & 3 & 50 & 2 & 22 & - & - & 3 & 50 & 5 & 71 \\
\hline Hospitalizações (Hp) & 2 & 17 & 3 & 30 & 2 & 33 & 4 & 44 & 3 & 43 & - & - & 3 & 43 \\
\hline Sintomatologia precoce (St) & - & - & - & - & 2 & 33 & - & - & 2 & 29 & 1 & 17 & 2 & 29 \\
\hline Dificuldades sensoriais (Ds) & 2 & 17 & 2 & 20 & 2 & 33 & 1 & 11 & 1 & 14 & 2 & 33 & 1 & 14 \\
\hline Baixa auto-estima (Be) & 1 & 8 & - & - & - & - & - & - & - & - & - & - & - & - \\
\hline Desempenho escolar pobre (De) & 1 & 8 & 3 & 30 & 2 & 33 & 2 & 22 & 5 & 71 & 3 & 50 & - & - \\
\hline Gravidez na adolescência (Ga) & - & - & - & - & 1 & 17 & 1 & 11 & 1 & 14 & - & - & 2 & 29 \\
\hline Bebê não desejado (Bñ) & 2 & 17 & 1 & 10 & 2 & 33 & 1 & 11 & 1 & 14 & - & - & 2 & 29 \\
\hline Criança adotada $(\mathrm{Ca})$ & - & - & - & - & - & - & - & - & - & - & - & - & 1 & 14 \\
\hline $\begin{array}{l}\text { Dificuldades financeiras da } \\
\text { família (Df) }\end{array}$ & 2 & 17 & 1 & 10 & 1 & 17 & 1 & 11 & - & - & - & - & - & - \\
\hline Insegurança e indecisão (Is) & - & - & - & - & 1 & 17 & 1 & 11 & 1 & 14 & 1 & 17 & - & - \\
\hline Superproteção (Su) & - & - & - & - & 1 & 17 & 1 & 11 & 1 & 14 & 1 & 17 & - & - \\
\hline Indiferença e rejeição (Ir) & 1 & 8 & 1 & 10 & 1 & 17 & 1 & 11 & - & - & - & - & - & - \\
\hline Agressividade (Ag) & - & - & - & - & - & - & 1 & 11 & - & - & - & - & - & - \\
\hline Depreciação (Dp) & 1 & 8 & - & - & - & - & - & - & - & - & - & - & - & - \\
\hline
\end{tabular}


Nota-se que as condições adversas mais freqüentemente associadas a problemas congênitos referem-se a problemas de saúde física da mãe na gestação $(50 \%)$ e de saúde física da criança (42\%). Associados aos problemas de saúde física da mãe durante a gestação apareceram, com mais freqüência, os problemas congênitos $(60 \%)$ e problemas de saúde física da criança (50\%). A ocorrência de alguma complicação no parto foi mais freqüentemente associada a problemas congênitos, problemas de saúde física da criança $\mathrm{e}$ crises convulsivas $(50 \%)$. Os fatores de risco que, com mais freqüência, apareceram relatados nas histórias das crianças com problemas de saúde física foram os problemas congênitos e os problemas de saúde física da mãe durante a gestação (55\%) e os episódios de hospitalização (44\%). Já nas crianças que manifestaram crises convulsivas, o desempenho escolar pobre foi o fator mais freqüente $(71 \%)$, seguido dos problemas de saúde física da mãe na gestação, complicações no parto e hospitalização (43\%).

A história de vida da criança que necessitou de hospitalização continha, com maior freqüência, o relato de problemas de saúde $(67 \%)$, crises convulsivas, desempenho escolar pobre e problemas de saúde física da mãe durante a gestação (50\%). Finalmente, considerando-se o desempenho escolar pobre como um fator potencialmente de risco para problemas emocionais e comportamentais, constatou-se que as condições adversas que mais freqüentemente a ele se associavam foram: crises convulsivas $(71 \%)$, hospitalização e problemas de saúde física da mãe durante a gestação $(43 \%)$.

\section{DISCUSSÃO}

Como se pode observar, o atraso no desenvolvimento aparece como única queixa que motivou a procura de atendimento, em $41 \%$ dos casos, e, nos demais, são relatadas outras dificuldades da criança, sendo mais freqüentes os problemas de fala, coordenação motora, aprendizagem e agitação. É interessante ressaltar que a maioria das crianças do grupo com queixa única tem idades inferiores a três anos (89\%) e as do grupo com queixa múltipla têm idades entre seis e oito anos (77\%). Quando a criança é pequena, a preocupação dos pais parece mais centrada no atraso geral do desenvolvimento do filho, sinalizado pela sua dificuldade em cumprir determinadas tarefas características de sua faixa etária. A procura de atendimento pelos pais, para a maioria dessas crianças, envolve a busca de orientação, visando a estimulação precoce do seu desenvolvimento. Já para as crianças maiores (seis a oito anos), a queixa dos pais é geralmente múltipla, provavelmente devido ao fato de a criança se encontrar em idade escolar e apresentando problemas de funcionamento, decorrentes do atraso, nas áreas de linguagem, motora e cognitiva, que interferem com a aquisição de novas habilidades e mesmo com o seu rendimento acadêmico. O desempenho escolar pobre, presente na vida da grande maioria das crianças, nas idades de seis a oito anos, pode se constituir também em um fator de risco para o aparecimento de problemas de ordem emocional e comportamental.

Os resultados da análise das circunstâncias adversas, presentes na história de vida desse grupo de crianças com atraso no desenvolvimento, deixam claramente evidente que a maioria das crianças foi exposta a três ou mais fatores de risco e que, além disso, em todas elas, se constatou a presença de uma ou mais das condições de risco biológico, sendo as mais freqüentes (acima de $30 \%$ ) problemas congênitos, problemas de saúde física da mãe durante a gestação, problemas de saúde física da criança, crises convulsivas, complicações no parto e hospitalização. Por outro lado, quando se procurou verificar que outros fatores apareciam mais frequientemente associados a cada um destes, o que se constatou foi a associação com fatores da mesma natureza, evidenciando a presença cumulativa do risco biológico que poderia aumentar a vulnerabilidade da criança a problemas de desenvolvimento. Uma outra condição de risco identificada freqüentemente nesta amostra refere-se ao desempenho escolar pobre que, por sua vez, também apareceu mais frequientemente associado com fatores de natureza biológica ,ou seja, crises convulsivas, hospitalização e problemas de saúde física da mãe na gestação. Parece então evidente a interligação entre atraso no desenvolvimento, condições biológicas adversas e desempenho escolar pobre.

Quando se procurou verificar que outros fatores de risco, além do biológico, apareceram na vida das crianças, o que se encontrou foi que $36 \%$ havia sido exposta a fatores do contexto ambiental tais como bebê não desejado, dificuldades financeiras da família, gestação na adolescência, criança adotada, insegurança elou inconsistência dos pais, superproteção, indiferença/rejeição, agressividade e depreciação. Pode-se dizer que muitos desses fatores já indicam diretamente a presença de condições de risco do tipo proximal (insegurança, superproteção, indiferença/rejeição, 
agressividade e depreciação) ou de condições que podem dificultar a interação dos pais com a criança, como pode ser o caso, por exemplo, de bebê não desejado e gestação na adolescência. A literatura tem amplamente apontado que estes fatores proximais se constituem em condições que podem maximizar os efeitos do risco biológico ${ }^{3,4,6}$. Nossos dados deixam evidente a presença de múltiplas condições de risco na história de vida das crianças com atraso no desenvolvimento, indo de encontro à consideração de Allen ${ }^{11}$ de que crianças com fatores de risco múltiplo, geralmente, têm um risco muito maior para problemas de desenvolvimento do que aquelas expostas a um único fator.

Embora seja comum encontrar, na literatura, estudos que incluem, dentre as condições de risco para atraso no desenvolvimento, a análise das variáveis ambientais, principalmente as relativas às condições de vida das famílias, na amostra aqui pesquisada não se levantarem informações suficientes para caracterizar o status sócio-econômico deste grupo. No entanto, poder-se-ia inferir, a partir das informações acerca do nível de escolaridade dos pais, que a maioria das crianças não provêm de famílias de nível sócio econômico tão prejudicado, dado que $73 \%$ dos pais e $64 \%$ das mães têm nível secundário ou superior completo. É bem possível que o grau de instrução dos pais tenha sido um fator que favoreceu a busca de serviços especializados para a criança.

Quando se atenta, na amostra aqui pesquisada, para a fonte de encaminhamento das crianças para o serviço de atendimento psicológico, nota-se que ela se constitui basicamente por profissionais da área médica (82\% dos casos), principalmente geneticistas e neurologistas. É possível que a presença do risco biológico tenha mobilizado, inicialmente, os pais a procurarem serviços médicos e que estes, por sua vez, passaram a servir como mediadores para o acesso da criança e da família a serviços de natureza psicológica. Por outro lado, é possível que a presença marcante de fatores de risco biológico, na amostra estudada, esteja, em parte, relacionada ao fato de o encaminhamento ter sido feito por profissionais da área médica. Estas considerações permitem o levantamento da seguinte questão: a probabilidade de atendimento especializado da criança com atraso no desenvolvimento e que tem presente em sua história de vida a condição de risco biológico é maior do que a da criança sem a presença dessa condição? Adams et al. ${ }^{7}$, p.380 discutem os resultados de seu trabalho sob este prisma: "nossos dados sugerem que, na região de Baton Rouge, as crianças que nascem com fatores de risco biológico têm maior probabilidade de receber serviços de intervenção do que as crianças sócio culturalmente prejudicadas". Os autores enfatizam que as crianças pertencentes ao grupo de risco duplo (biológico e social) apresentam um índice maior de acesso a programas de intervenção precoce do que as crianças dos grupos de baixo risco ou do grupo de risco social. Eles defendem que, do ponto de vista preventivo, as crianças saudáveis pertencentes ao grupo de risco social e aquelas com risco biológico, com ou sem o risco social, deveriam se beneficiar dos programas de intervenção. Já Allen, $1993^{11}$, considera que poderão ser tomadas diferentes decisões com relação à indicação de serviços de apoio para as crianças de risco. Assim, crianças de múltiplos riscos, facilmente identificadas, devem ser encaminhadas para serviços de estimulação, enquanto que crianças com menos riscos devem ser identificadas e acompanhadas. As crianças que demonstrarem atrasos no desenvolvimento devem ser encaminhadas para avaliações específicas e serviços terapêuticos. Para qualquer um dos casos, suportes sociais específicos podem ser necessários, dependendo das mudanças que ocorrerem com a criança ou com a família. Deste modo, crianças pertencentes a diferentes grupos de risco deverão ter seu desenvolvimento monitorado sistematicamente, através de avaliações periódicas que possibilitem a intervenção, tão logo seja julgada necessária. Tomando como exemplo, as crianças diagnosticadas precocemente como portadoras de hipotireoidismo congênito ou de fenilcetonúria deveriam receber atenção com relação ao acompanhamento nas diferentes áreas do desenvolvimento, possibilitando outras intervenções, além da médica. Isto também se aplica às crianças expostas a várias outras condições de risco (prematuridade, baixo peso ao nascer, anoxia neonatal, desnutrição, dentre outras).

Além disso, condições precárias de vida, de saúde e de educação não só podem agir diretamente sobre a criança, como podem também limitar seu acesso a serviços especializados e desencadear eventos estressantes para as famílias, comprometendo a interação dos pais com a criança e formando uma cadeia de risco para o desenvolvimento. Nesse sentido, serviços de atenção primária à saúde também devem ter como preocupação o acompanhamento do desenvolvimento geral da criança, $o$ atendimento e orientação a gestantes e mães, enfatizando a importância do cuidado e de uma interação saudável com a criança.

Numa perspectiva ideal, os serviços deveriam estruturar estratégias que possibilitassem localizar 
crianças em condição de risco, acompanhar seu desenvolvimento e informar os membros da comunidade sobre a existência destes serviços numa tentativa de promover a prevenção nos níveis primário e secundário, minimizando os efeitos do risco e diminuindo a necessidade de intervenções no nível terciário.

\section{CONCLUSÕES}

1- O risco biológico se fez presente em todas as crianças da amostra aqui estudada.

2- A presença do risco biológico, aliada ao nível educacional dos pais, (predominantemente médio e su- perior) parece ter facilitado o acesso a serviço especializado.

3- Profissionais da área médica têm a possibilidade de identificar crianças do grupo de risco (especialmente do biológico) e devem ser conscientizados da necessidade de encaminhamento a serviços que possibilitem monitoramento do desenvolvimento destas crianças.

4- Faz-se necessária a conscientização de diferentes setores da comunidade com relação aos benefícios da identificação precoce das crianças de risco biológico ou ambiental e posterior acompanhamento de seu desenvolvimento.

GRAMINHA SSV \& MARTINS MA de O. Adverse conditions in the life of children with developmental retardation. Medicina, Ribeirão Preto, 30: 259-267, abr./june 1997.

ABSTRACT: The objective of the present study was to identify the risk factors to which children with delayed development referred to the Center of Applied Psychology of FFCLRP-USP over the last five years had been exposed. The group consisted of 12 boys and 10 girls ranging in age from 1 month to 8 years. The data were extracted from the interviews held with the mothers as the time of child enrollment in the service. The problems reported by de mothers on the occasion of the complaint and the adverse events and circumstances that ocurred in the child's life were classified according to the Category System elaborated by the authors of the present study. The results showed that, in addition to retardation, the most frequent problems reported on the occasion of the complaint were speech, motor coordination, learning and agitation/restlessness problems. Analysis of the adverse circumstances in the life history of this group of children demonstrated that all of them had been exposed to one or more biological risk conditions, the most frequent being congenital problems, mother's physical health problems during pregnancy, child's physical health problems, convulsive seizures, complications of delivery, and hospitalization. Furthermore, $36 \%$ of the children had been exposed to environmental risk factors, especially those linked to inadequate attitudes and practices of care and education. On the basis of the results obtained, we discuss the implications of the presence of biological risks as an important condition for access to specialized services on the part of the children and their families.

UNITERMS: Risk Factors. Biological Factors. Child Development.

\section{REFERÊNCIAS BIBLIOGRÁFICAS}

1 - HANSON MJ \& LYNCH EW. Early intervention. PRO-ED, Austin, 1989.

2 - KORHONEN TT et al. Neuropsychological sequelae of perinatal complications: a six-year follow-up. J Clin Psychol 22: 226-235, 1993.

3 - BENDERSKY M \& LEWIS M. Environmental risk, biological risk and developmental outcome. Dev Psychol 30: 484-494, 1994.

4 - CENSULLO M. Developmental delay in healthy premature infants at age two years: implications for early interventions. $\mathbf{J}$ Dev Behav Pediatr 15: 99-104, 1994.

5 - SMITH L; ULVUND SE \& LINDEMANN R. Very low birth weight infants (<1501) at double risk. J Dev Behav Pediatr 15: 7-13, 1994.

6 - WERNER EE. A longitudinal study of perinatal risk. In: FARRAN DC \& MCKINNEY JD, eds. Risk in intellectual and psychosocial development. Academic Press, New York, p. 3-27, 1986.
7 - ADAMS CD; HILLMAN N \& GAYDOS JR. Behavioral difficulties in toddlers: impact of sociocultural and biological risk factors. J Clin Child Psychol 23: 373-381, 1994.

8 - THOMPSON RJ et al. Developmental outcome o very low birth weight infants as a function of biological risk and psychsocial risk. J Dev Behav Pediatr 15: 232-238, 1994.

9 - GRAMINHA SSV \& MARTINS MAO. Procura de atendimento psicológico para crianças: características da problemática relatada pelos pais. PSICO 25: 53-79,1994.

10 - GRAMINHA SSV; MARTINS MAO \& MIURA RT. Fatores potenciais de risco em crianças com problemas emocionais/comportamentais. PSICO 27:153-175,1996.

11 - ALLEN MC. The high risk infant. Pediatr Clin North Am 40: 479-490, 1993.

Recebido para publicação em 20/09/96

Aprovado para publicação em 07/05/97 